\title{
Knockdown of nuclear factor erythroid 2-related factor 2 by lentivirus induces differentiation of glioma stem-like cells
}

\author{
JIANHONG ZHU $^{1}$, HANDONG WANG ${ }^{1}$, YOUWU FAN ${ }^{1}$, YANGCHUN HU ${ }^{1}$, \\ XIANGJUN JI $^{1}$, QING SUN ${ }^{1}$ and HUANDONG LIU ${ }^{2}$ \\ ${ }^{1}$ Department of Neurosurgery, Jinling Hospital, Medical School of Nanjing University, Nanjing, Jiangsu 210002; \\ ${ }^{2}$ Department of Neurosurgery, Southern Medical University, Guangzhou, Guangdong 510515, P.R. China
}

Received March 14, 2014; Accepted June 6, 2014

DOI: $10.3892 / o r .2014 .3320$

\begin{abstract}
Glioma stem cells (GSCs) are key in the progression and recurrence of glioblastoma. Inducing the differentiation of GSCs is an important therapeutic target for glioblastoma. Nuclear factor erythroid 2-related factor 2 (Nrf2) has been reported to be important in maintaining the stem cell status of GSCs; however, its association with differentiation has not been studied. Herein, we knocked down Nrf2 from GSCs to investigate the role of Nrf2 in the differentiation of GSCs. First, Nrf2 expression was observed at different stages of differentiation; then, Nrf2 was knocked down and the association of Nrf2 with differentiation degree was observed in vitro. Finally, GSCs were planted in nude mice to study the association of Nrf2 with differentiation in vivo. The expression of Nrf2 decreased with the differentiation process. Following Nrf2 knockdown, the proportion of sphere-like colonies decreased and the dendritic cells in spheres increased; the expression of Nrf2 significantly decreased while the expression of differentiation marker glial fibrillary acidic protein (GFAP) and $\beta$ III-tubulin increased both at the protein and the gene level. In the xenografts of nude mice, the differentiation of tumor cells was improved. These results suggest that Nrf2 is a key factor inhibiting the differentiation of GSCs, and knockdown of Nrf2 may promote the differentiation process, providing a therapy target for GSCs.
\end{abstract}

\section{Introduction}

Glioblastoma is a type of lethal brain tumor with a median survival of approximately 14 months, even with aggressive surgery, radiation and chemotherapy $(1,2)$. Malignant gliomas have a highly tumorigenic subpopulation, known as cancer stem cells (CSCs), which drives tumor formation and proliferation (3-5). These cells are defined as glioma stem cells

Correspondence to: Dr Handong Wang, Department of Neurosurgery, Jinling Hospital, Medical School of Nanjing University, 305 East Zhongshan Road, Nanjing, Jiangsu 210002, P.R. China E-mail: njhdwang@hotmail.com

Key words: glioma stem cells, glioblastoma, Nrf2, differentiation
(GSCs) with higher proliferation and poorer differentiation than glioblastoma cells around them (6-10). Although these cells represent only a small fraction of the tumor bulk, their high self-renewal capacity is thought to sustain tumor growth (11). Therapies which induce the differentiation of GSCs to non-stem-like glioblastoma cells can decrease the growth of tumor and improve the sensitivity to radiotherapy and chemotherapy $(3,11,12)$. For this reason, differentiation therapy is regarded as a therapeutic strategy for GSCs. Inducing the differentiation of GSCs will be valuable for patients suffering from glioblastoma.

Nuclear factor erythroid 2-related factor $2(\mathrm{Nrf} 2)$ is a member of the cap ' $n$ ' collar family, which has been described as a central orchestrator of the expression of antioxidant and detoxifying genes $(13,14)$. Nrf2 escapes Kelch-like ECH-associating protein 1 (Keap1) and accumulates in the nucleus where it binds to antioxidant response element (ARE) sequences in the regulatory regions of its target genes to induce their expression (14-16).

Recently, the role of Nrf2 was observed in several types of tumors, such as non-small cell lung cancer, glioma, bladder carcinoma and hepatocarcinoma $(17,18)$. Nrf2 protects tumors from chemotherapy and radiotherapy $(19,20)$. Our previous study found the significant role of Nrf2 in the self-renewal of GSCs (21). Furthermore, knockdown of Nrf2 significantly reduced the expression of stem cell markers Bmil, Sox2 and Cyclin E (21). Since GSCs were generally at a poor stage of differentiation and performed high proliferation and low maturity, we hypothesized that Nrf2 plays an important role in the differentiation of GSCs, and inhibiting Nrf2 could induce GSC differentiating to non-stem-like glioblastoma cells, decreasing the growth of the tumor and improving the sensitivity to radiotherapy and chemotherapy.

In this study, the GSCs were obtained from the tissues of patients with glioblastoma and Nrf2 was knocked down by lentivirus-transported shRNA to investigate the role of Nrf2 in the differentiation of GSCs both in vitro and in vivo, providing a possible therapeutic target for GSCs.

\section{Materials and methods}

The study was approved by the Ethics Committee of Jinling Hospital (Nanzi 20120017). Patients who were recruited 
provided written informed consent allowing the scientific use of their samples. Animal experiments were approved by the Animal Ethics Committee of the Animal Experiment Center at Jinling Hospital (SCXK 2012-012).

The GSCs used in this study were separated from glioblastoma tissue obtained from patients. The experiment was divided into three parts: i) induction of the differentiation of primary spheres and analysis of the expression of Nrf2 during the differentiation, ii) knockdown of Nrf2 from GSCs by lentivirus and examination of the differentiation in vitro, iii) planting of the GSCs in nude mice and studying the differentiation in vivo. The experimental process is shown in Fig. 1.

Patient glioma samples. Glioma specimens were obtained from the Department of Neurosurgery in Jinling Hospital. All the patients were diagnosed with glioma. The tumors were classified as Stage IV by two pathologists, according to the WHO Classification of central nervous system tumors.

Primary sphere culture. Tumors were dissociated with $0.25 \%$ trypsin and released by gentle pipetting and filtrated through a $70-\mu \mathrm{m}$ cell strainer. Adherent culture of cells was performed by plating the cells in a gelatin-coated plastic flask in DMEM for $24 \mathrm{~h}$ and washing with phosphate buffered saline (PBS) to remove red blood cells and cell debris. Then, the tumor cells were collected and seeded in neural stem cell (NSC) medium (Gibco-BRL, USA) at a density of 2,000 cells $/ \mathrm{cm}^{2}$ to obtain floating tumor-spheres. Primary GSCs were incubated at $37^{\circ} \mathrm{C}$ in an atmosphere containing $5 \% \mathrm{CO}_{2}$ for 5-7 days. The medium was half-renewed every 3 days. After growing $>100 \mu \mathrm{m}$ in diameter, the spheres were tentatively defined as GSC spheres.

Induction of differentiation of primary spheres. To induce differentiation, primary spheres were reseeded into a 24-well cluster at a density of approximately 50 spheres/well. Cells were cultivated in DMEM/F12 (Gibco-BRL) and supplemented with $10 \%$ fetal bovine serum (FBS). Cells were separated into 6 equal groups, each group containing 4 wells. Approximately $4 \mathrm{~h}$ later, spheres in every well adhered to the plates and dendritic branches could be found at the base. Twelve hours, 24 h, 2, 3 and 5 days after induction, we chose 2 wells in each group to fix with formaldehyde, respectively. We collected one more well of each group to prepare protein and 1 well to stain for immunocytochemistry. Seven days later, we separated each group into 2 equal parts: part 1 was observed with microscopy and part 2 was stained with gentian violet. They were viewed with a microscope (Carl Zeiss, Germany).

Knockdown of Nrf2 by lentivirus and observation of the morphology of differentiation in spheres in vitro. Primary spheres were dissociated with Accutase (Sigma-Aldrich, USA) for $15 \mathrm{~min}$ and equal cells were reseeded into 6-well plates $\left(1 \times 10^{4}\right.$ cells/well). Lentiviruses for expression of scrambled shRNA or Nrf2 shRNA were diluted in NSC medium containing $5 \mu \mathrm{g} / \mathrm{ml}$ polybrene, and the medium was added to GSCs according to the groups separately. After $72 \mathrm{~h}$, infected cells rebuilt secondary spheres and were selected for puromycin resistance by refeeding the cells with fresh NSC medium containing $5 \mu \mathrm{g} / \mathrm{ml}$ puromycin for $24 \mathrm{~h}$.
Then, the spheres were reseeded into 6-well plates. Twenty-four hours after reseeding, spheres in half of the wells were collected for protein and mRNA, and the other half were collected for morphology analysis. At $24 \mathrm{~h}$ and 3 days after reseeding, the sphere-like colonies in the scrambled and $\mathrm{Nrf} 2$ shRNA-treated cells were scored in 20 random $4 \mathrm{x}$ fields by two independent scorers who were unaware of the sample designation.

The human Nrf2 short hairpin RNA (shRNA) sequence was: 5'-GCAGTTCAATGAAGCTCAACT-3', while the scrambled shRNA sequence was: 5'-TTCTCCGAACGTGTCACGT-3'. The lentivirus vector was purchased from GenePharma (Shanghai, China).

Western blotting. Equal amounts of proteins were separated using SDS-PAGE (8-12\% gradient gel). Separated proteins were transferred onto PVDF membranes (Millipore, Germany) and each membrane was cut into narrow pieces according to the protein molecular massive marker (Thermo, USA), blocked with $5 \%$ non-fat milk for $1 \mathrm{~h}$ at room temperature and probed with the appropriate antibody, as described previously. The dilutions of primary and secondary antibody were made in 3\% BSA and Tris-buffered saline (TBST) with $0.1 \%$ Tween-20, respectively. The membranes were incubated in primary antibody overnight at $4^{\circ} \mathrm{C}$, and in secondary antibody for $60 \mathrm{~min}$ at room temperature. The primary antibodies used were against: Nrf2 (1:1,000; Abcam), glyceraldehyde3-phosphate dehydrogenase (GAPDH; 1:5,000; Bioworld, USA), glial fibrillary acidic protein (GFAP; 1:500; BD, USA), BIII-tubulin (1:10,000; Abcam). The secondary antibody was the anti-rabbit-IgG-HRP antibody conjugated (Bioworld), and it was used at a 1:5,000 dilution.

RNA isolation and quantification. GSCs were non-infected or infected with lentiviruses that expressed either scrambled or Nrf2 shRNA, as described above. At $72 \mathrm{~h}$ after transduction, RNA was isolated from cells. RNA isolation and cDNA was synthesized using Strand cDNA synthesis Kit (Takara, Japan). RNA was isolated from three independent cell culture preparations. Levels of transcripts for specific genes were determined by SYBR-Green quantitative reverse transcription-PCR (qRT-PCR), using gene-specific primers for human transcripts encoding Nrf2, NAD(P)H:quinone oxidoreductase-1 (NQO-1), heme oxygenase-1 (HO-1), cyclin E, $\beta I I I-t u b u l i n$, GFAP. Sequences of primers for the remaining human genes are shown in Table I.

Immunocytochemistry and immunofluorescence. At $24 \mathrm{~h}$ after seeding, cells were fixed with $4 \%$ formaldehyde (SigmaAldrich, USA) in PBS for $20 \mathrm{~min}$ at room temperature, and permeabilized in $0.1 \%$ Triton $\mathrm{X}-100$ in PBS for $10 \mathrm{~min}$ at room temperature. Then, cells were blocked using $5 \%$ bovine serum albumin (BSA; Sigma-Aldrich) in PBS for $1 \mathrm{~h}$ at room temperature. Following blocking, cells were incubated in $\mathrm{Nrf2}$, GFAP and $\alpha$-tubulin primary antibody overnight, at $4^{\circ} \mathrm{C}$ on a rocking platform. Cells were washed 3 times in PBS. The appropriate secondary antibody was then added and allowed to incubate for $1 \mathrm{~h}$ in the dark at room temperature. Images of the cells were captured with a fluorescence microscope (Carl Zeiss). Primary antibodies used were against: Nrf2 (1:100; 


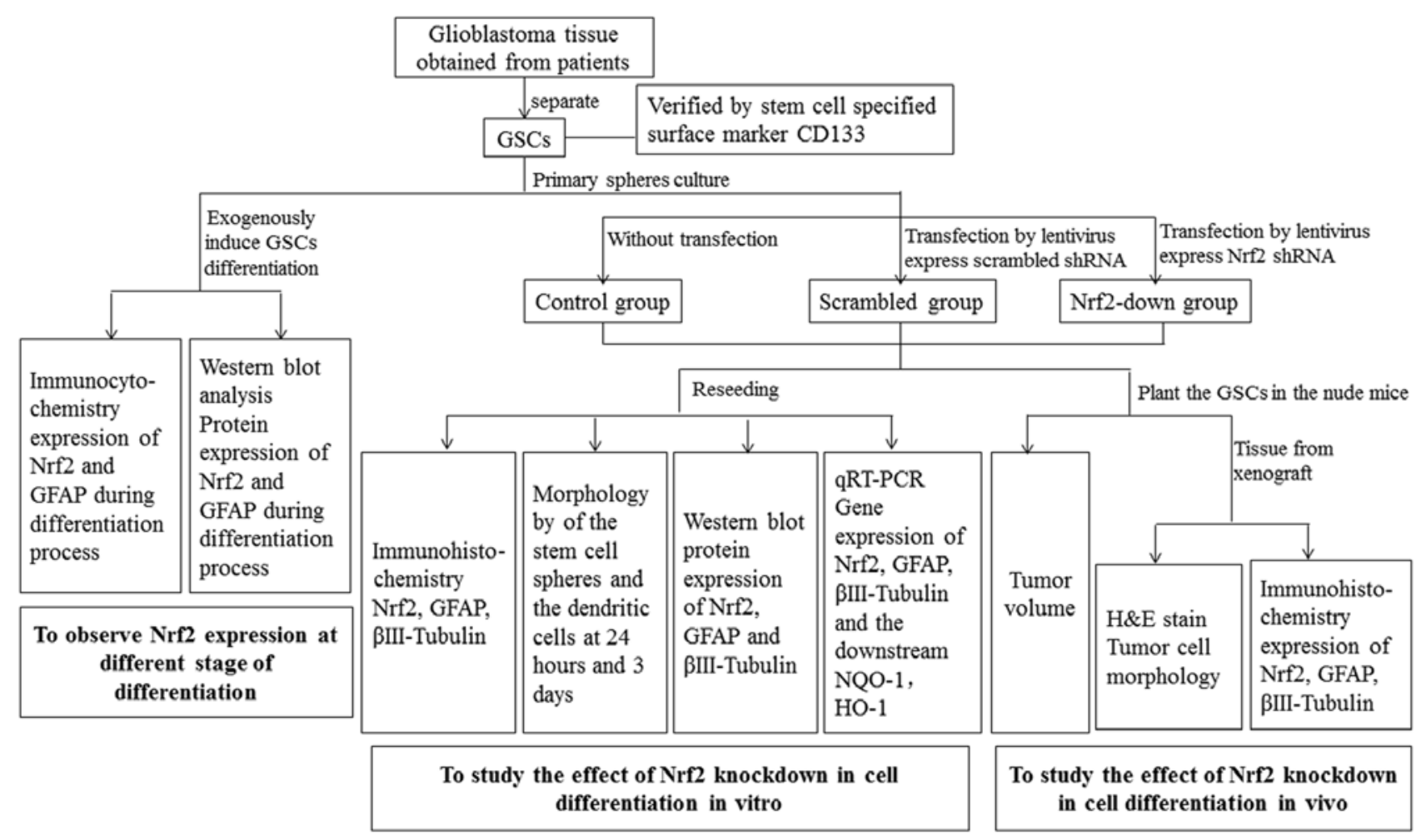

Figure 1. Flow process diagram of this study. GSCs, glioblastoma stem cells; Nrf2, nuclear factor erythroid 2-related factor 2; GFAP, glial fibrillary acidic protein; shRNA, short hairpin RNA; NQO-1, NAD(P)H:quinone oxidoreductase-1; HO-1, heme oxygenase-1; qRT-PCR, quantitative reverse transcriptionPCR; H\&E, hematoxylin and eosin.

rabbit polyclone; Abcam), GFAP (1:100; rabbit monoclone; BD), $\beta$ III-tubulin (1:1,000; rabbit monoclone; Abcam), CD133 (1:100; rabbit polyclone; Biobyte). Secondary antibodies were against mouse-IgG-FITC (Sigma-Aldrich), rabbit-IgGCy3 (F0382; Sigma-Aldrich). Nuclei were visualized using 4',6-diamidino-2-phenylindole (DAPI) staining (D9542; Sigma-Aldrich) at 1:10,000 in PBS.

Knockdown of Nrf2 by lentivirus and observation of the differentiation in vivo. Lentiviruses for expression of scrambled shRNA or Nrf2 shRNA were diluted in NSC medium containing $5 \mu \mathrm{g} / \mathrm{ml}$ polybrene, and the medium was added to GSCs according to the groups separately. After $72 \mathrm{~h}$, infected cells were selected for puromycin resistance by refeeding the cells with fresh NSC medium containing $5 \mu \mathrm{g} / \mathrm{ml}$ puromycin for $24 \mathrm{~h}$.

Spheres were then dissociated with Accutase (Sigma-Aldrich) for $15 \mathrm{~min}$ and equal cells $\left(2 \times 10^{4}\right)$ were dissociated in $50 \mu \mathrm{l}$ PBS and implanted subcutaneously into the flanks of 4-week-old male nude mice (6 mice in each group, a total of 3 groups, mice were randomly selected). Five weeks later, animals were sacrificed and xenograft tumors were measured and subjected to immunofluorescent analysis. Tumor growth was measured using an external caliper every week and tumor volume was calculated as (length/2) $\mathrm{x}$ width $^{2}$. After sacrifice, the tumors were removed for paraffin sectioning. The sections were stained by hematoxylin and eosin (H\&E), and treated with polyclonal rabbit antibodies to Nrf2, GFAP, $\beta$ III-tubulin. For xenografts of nude mice, secondary antibody was the anti-rabbit-IgG-HRP antibody conjugated (1:5,000 dilution; Bioworld) for $1 \mathrm{~h}$, and stained with 3,3'-diaminobenzidine tetrahydrochloride (DAB).
Table I. Sequences of primers for the remaining human genes.

\begin{tabular}{ll}
\hline Gene & \multicolumn{1}{c}{ Primer sequence } \\
\hline Nrf2 & F: 5'-TCAGCGACGGAAAGAGTATGA-3' \\
& R: 5'-CCACTGGTTTCTGACTGGATGT-3' \\
GAPDH & F: 5'-GAAATCCCATCACCATCTTC-3' \\
& R: 5'-CCACTGGTTTCTGACTGGATGT-3' \\
ßIII-tubulin & F: 5'-CAAGATGTCGTCCACCTTCAT-3' \\
& R: 5'-CTCAGACACCAGGTCGTTCAT-3' \\
Cyclin E & F: 5'-ACCAGTTTGCGTATGTGA-3' \\
& R: 5'-TGTGGGTCTGTATGTTGTG-3' \\
GFAP & F: 5'-AGGGACAATCTGGCACAGG-3' \\
& R: 5'-CGGTAGTCGTTGGCTTCG-3' \\
HO-1 & F: 5'-TCTCCGATGGGTCCTTACACTC-3' \\
& R: 5'-GGCATAAAGCCCTACAGCAACT-3' \\
NQO-1 & F: 5'-ATGGTCGGCAGAAGAGC-3' \\
& R: 5'-GGAAATGATGGGATTGAAGT-3'
\end{tabular}

Nrf2, nuclear factor erythroid 2-related factor; GAPDH, glyceraldehyde-3-phosphate dehydrogenase; GFAP, glial fibrillary acidic protein; HO-1, heme oxygenase-1; NQO-1,NAD(P)H:quinone oxidoreductase- $1 ; \mathrm{F}$, forward; $\mathrm{R}$, reverse.

Statistical analysis. All statistical analyses were performed using SPSS 10.0 software (SPSS Inc, USA). Data are shown as means \pm SD. Data were statistically analyzed using one-way 
A
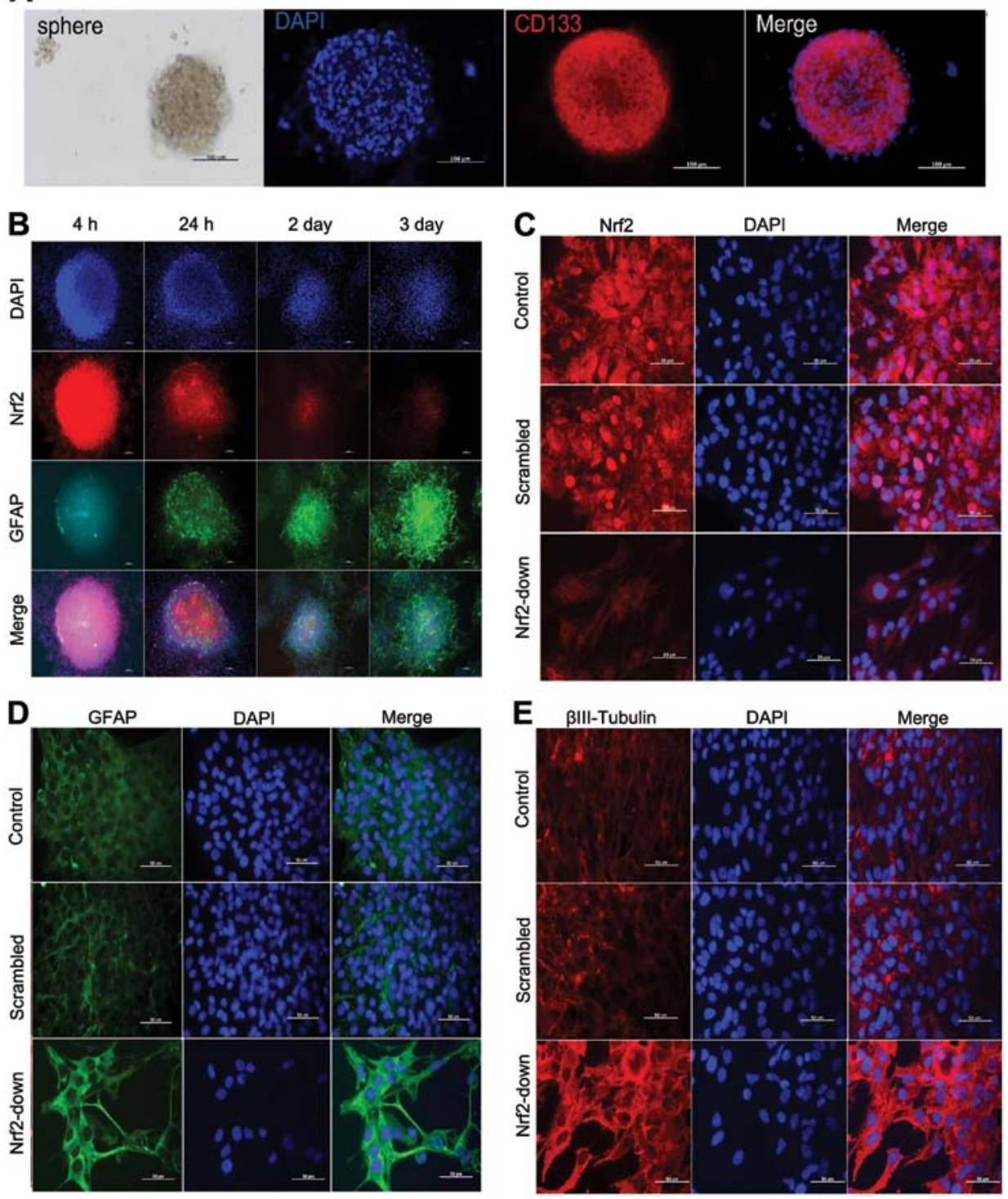

Figure 2. The expression of Nrf2 and differentiation markers in GSCs by immunofluorescence. (A) The primary spheres were identified with CD133 (red) in immunofluorescence. (B) The expression levels of differentiation marker GFAP substantially increased after FBS inducing differentiation, and the Nrf2 expression decreased during that process. (C) Immunofluorescence staining for Nrf2; the expression of Nrf2 decreased in the Nrf2-down group. (D) Immunofluorescence staining for GFAP; the expression of GFAP increased in the Nrf2-down group. (E) Immunofluorescence staining for $\beta I I I-t u b u l i n ;$ the expression of $\beta$ III-tubulin increased in the Nrf2-down group. Nuclear staining was with DAPI. Nrf2, nuclear factor erythroid 2-related factor 2; GFAP, glial fibrillary acidic protein; DAPI, 4',6-diamidino-2-phenylindole; GSCs, glioblastoma stem cells; FBS, fetal bovine serum.

analysis of variance (ANOVA). $\mathrm{P}<0.05$ was considered to indicate a statistically significant difference.

\section{Results}

The expression of Nrf2 decreases during the differentiation of primary GSCs and the GFAP increases. After reseeding, the primary GSC spheres were observed under microscopy (Fig. 2A). The primary spheres were identified with CD133 in immunocytochemistry and the high expression of CD133 in the cells was viewed easily (Fig. 2A).

After $24 \mathrm{~h}$ of differentiation induction, spheres in every well adhered to the plates and dendritic branches were found in the basement (Fig. 2B). The expression level of differentiation marker GFAP was substantially increased after FBS inducing differentiation. The expression level of Nrf2 was high in the base, and it decreased as cell differentiation increased (Fig. 3). These observations demonstrate the function of Nrf2 in the differentiation of glioma stem-like cells.

Knockdown of Nrf2 by lentivirus improves the differentiation of glioma stem-like cells in morphology. Twenty-four hours after reseeding, there were two types of sphere-like colonies in each group: i) the suspended spheres which floated in the medium and ii) the adhered spheres, in the basement of which were the dendrites of differential cells. Nrf2 knockdown increased the adhesion of spheres in GSCs (Fig. 4A). The proportions of adhered spheres in the control and scrambled group were $20.7 \pm 1.36$ and $20.5 \pm 3.41 \%$ respectively. However, the proportion of adhered spheres was $77.7 \pm 4.19 \%$ in the Nrf2down group (Fig. 4B). The percentage of adhered spheres was higher in the Nrf2-down group $(\mathrm{P}=0.001)$. 

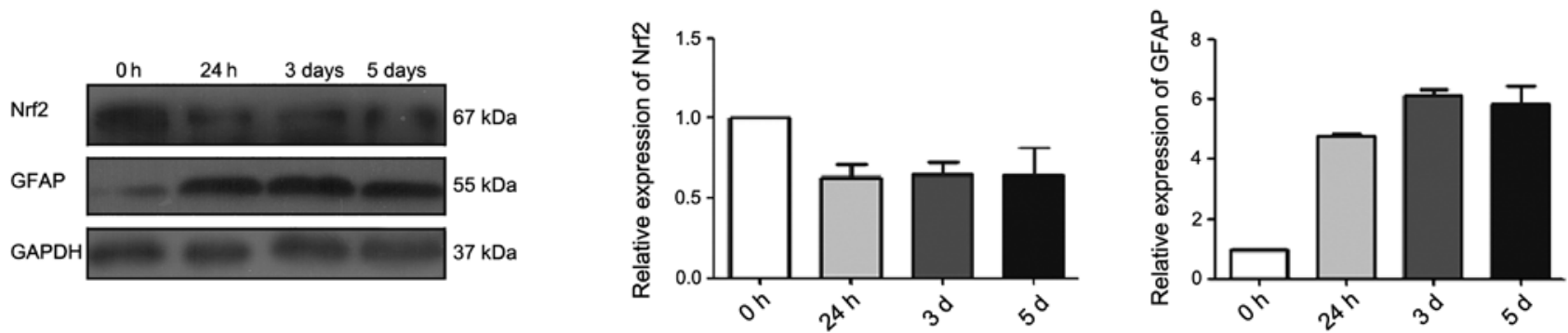

Figure 3. Western blotting of protein expression in GSCs during differentiation. Expression levels of differentiation marker GFAP substantially increased after FBS inducing differentiation and the Nrf2 level decreased. Data are shown as means \pm SD. Nrf2, nuclear factor erythroid 2-related factor 2; GFAP, glial fibrillary acidic protein; GAPDH, glyceraldehyde-3-phosphate dehydrogenase; GSCs, glioblastoma stem cells; FBS, fetal bovine serum.

A

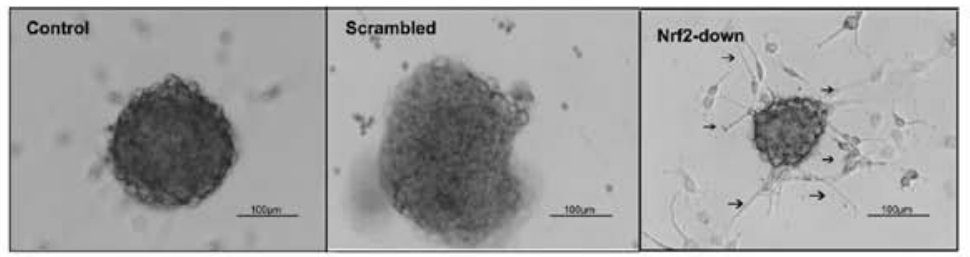

C

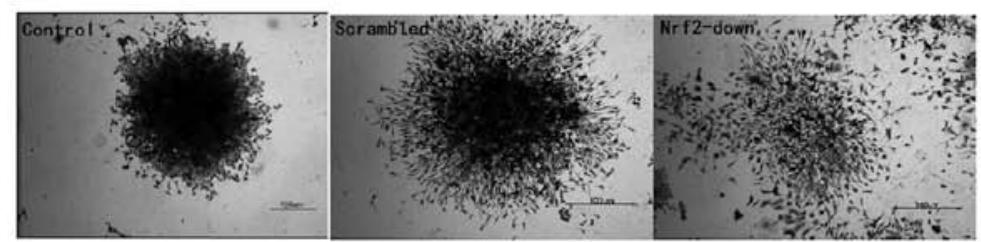

E

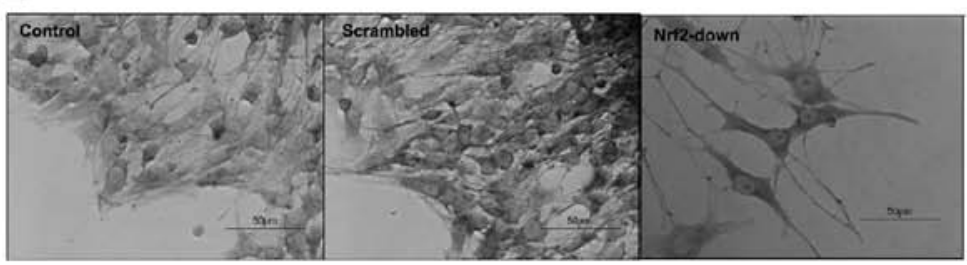

B

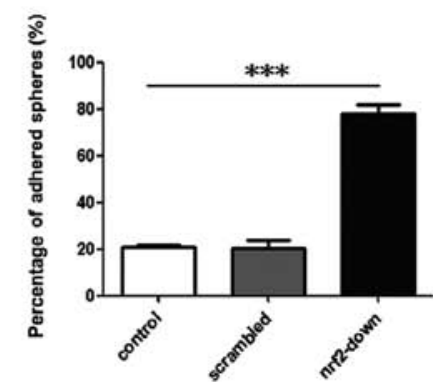

D ฮู

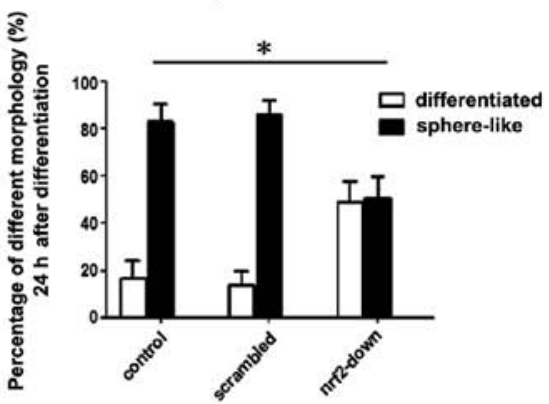

$\mathbf{F}$

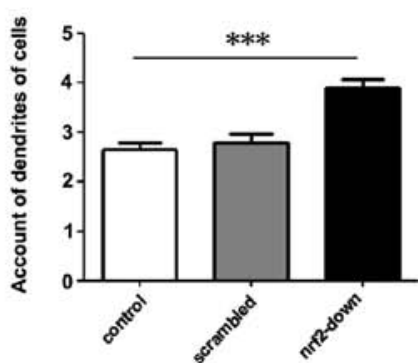

Figure 4. Morphology among different groups at $24 \mathrm{~h}$ and 3 days after reseeding. (A) The morphology of spheres at $24 \mathrm{~h}$ after reseeding. In the Nrf2-down group, there were dendrites of differential cells at the base. (B) The proportion of adhered spheres in each group. The proportion significantly increased in the Nrf2-down group. (C) Differentiated colonies of GSCs increased in the Nrf2-down group at 3 days after reseeding. (D) The percentage of differentiated colonies of GSCs increased in the Nrf2-down group. (E) More dendrites can be seen in the Nrf2-down group than in the control and the scrambled group. (F) The account of dendrites significantly increased in the Nrf2-down group compared to the control and the scrambled group. Data shown as means \pm SD. ${ }^{*} \mathrm{P}<0.05 ;{ }^{* * *} \mathrm{P}<0.005$. Nrf2, nuclear factor erythroid 2-related factor 2; GSCs, glioblastoma stem cells.

After 3 days, the majority of the GSCs differentiated in the Nrf2-down group. In the other groups, GSCs maintained the sphere-like and low differentiated colonies (Fig. 4C). GSCs in the control group and the group infected with the scrambled shRNA vector exhibited $17.6 \pm 2.69 \%$ and $13.9 \pm 2.23 \%$ ratios of differentiated colonies, whereas the ratio was $48.6 \pm 3.40 \%$ in the Nrf2-down group (Fig. 4D). There was also a higher number of differentiated colonies in the Nrf2-down group $(\mathrm{P}=0.018)$.
At the base of the spheres, there were many dendrites in adhered cells, which were the morphological markers of differentiation. In the Nrf2-down group, there were considerably more dendrites than in the control and scrambled group, and the dendrites were much longer (Fig. 4E). The average dendrites in the control and scrambled group were $2.65 \pm 0.67$ and 2.8 \pm 0.70 , and the dendrites in the Nrf2-down group were $3.9 \pm 0.72$ (Fig. 4F). The Nrf2-down group differentiated faster than the other groups in morphology $(\mathrm{P}<0.001)$. 
A
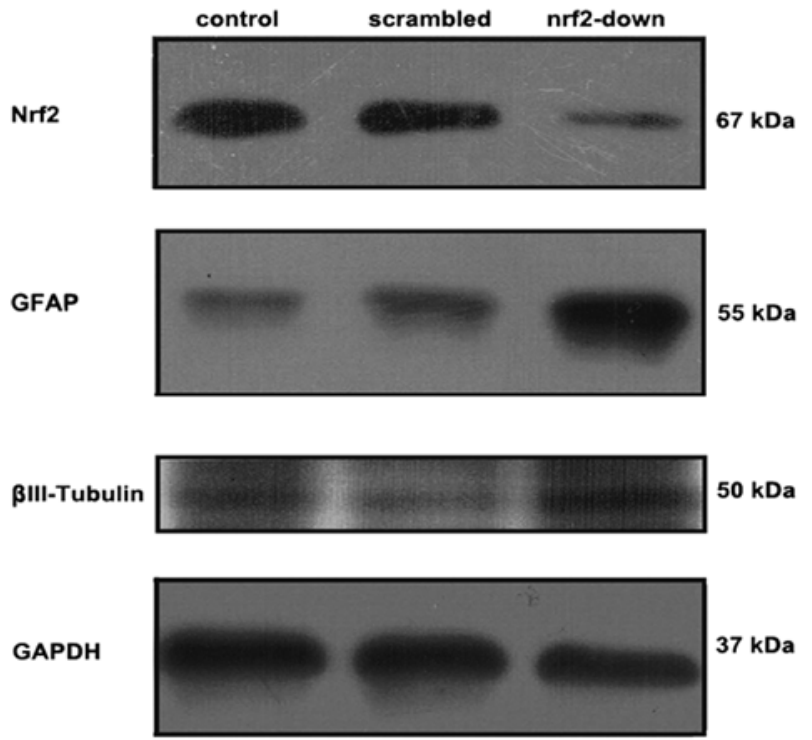

B
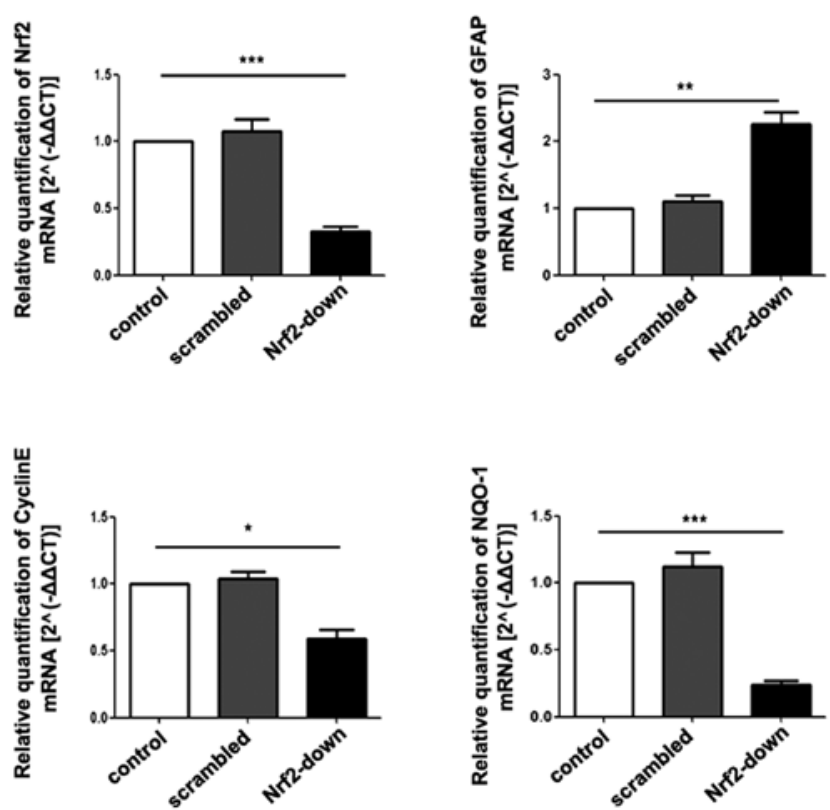
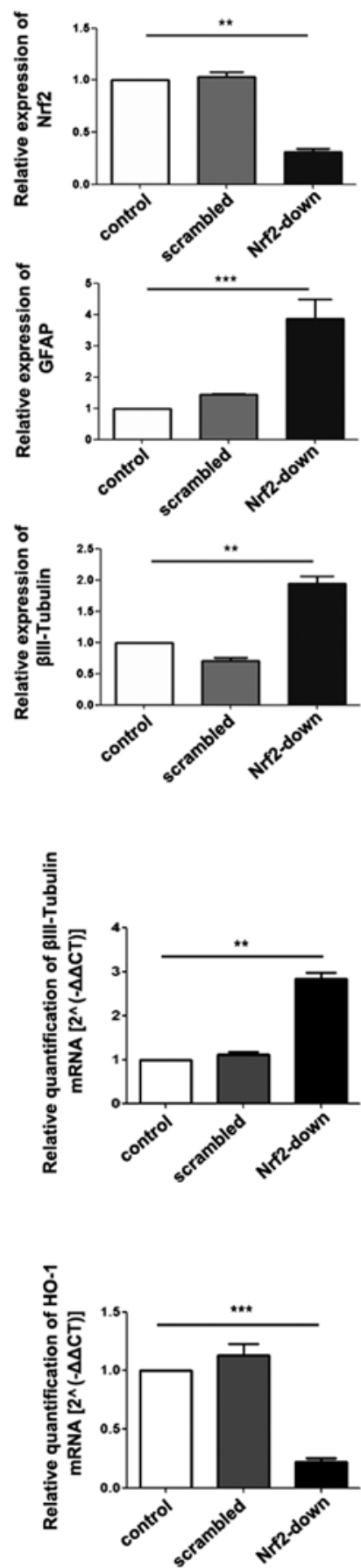

Figure 5. Protein and gene expression of Nrf2 and differentiation-related markers among different groups. Western blot analysis of proteins isolated from GSCs, protein expression of GFAP and $\beta$ III-tubulin increased in the Nrf2-down group. (B) RT-qPCR of RNA isolated from GSCs after transduction. GFAP and $\beta$ IIItubulin increased substantially with the knockdown of Nrf2, while Cyclin E, NQO-1 and HO- 1 decreased. Data are shown as means $\pm \mathrm{SD}$. ${ }^{*}<0.05$; ${ }^{* *} \mathrm{P}<0.01$; ${ }^{* * * *} \mathrm{P}<0.005$. Nrf2, nuclear factor erythroid 2-related factor 2; GFAP, glial fibrillary acidic protein; GAPDH, glyceraldehyde-3-phosphate dehydrogenase; NQO-1, NAD(P)H:quinone oxidoreductase-1; HO-1, heme oxygenase-1; GSCs, glioblastoma stem cells; qRT-PCR, quantitative reverse transcription-PCR.

Knockdown of Nrf2 by lentivirus induces the differentiation-associated markers. In western blot analysis and immunocytochemistry, Nrf2 expression was lower in the Nrf2-down group than in the other two groups (Fig. 5A and C), confirming that the Nrf2 was downregulated by lentivirus. Furthermore, the expression levels of differentiation marker GFAP and $\beta I I I-t u b u l i n$ protein increased after Nrf2 knockdown as demonstrated both by western blotting (Fig. 5A) and immunocytochemistry analysis (Fig. 2D and E).
Nrf2 knockdown in GSCs induces the expression of differentiation-associated genes. qRT-PCR demonstrated that there was a significant decrease in the gene expression of stem cell marker cyclin $\mathrm{E}$ after Nrf2 knockdown $(\mathrm{P}<0.001$; Fig. 5B). In contrast, gene expression of astrocyte marker GFAP $(\mathrm{P}=0.008)$ and neural surface marker $\beta$ III-tubulin $(\mathrm{P}=0.004)$ was significantly increased in the Nrf2-down group, which could be attributed to spontaneous differentiation within the cell population (Fig. 5B). Furthermore, the expression levels 
A

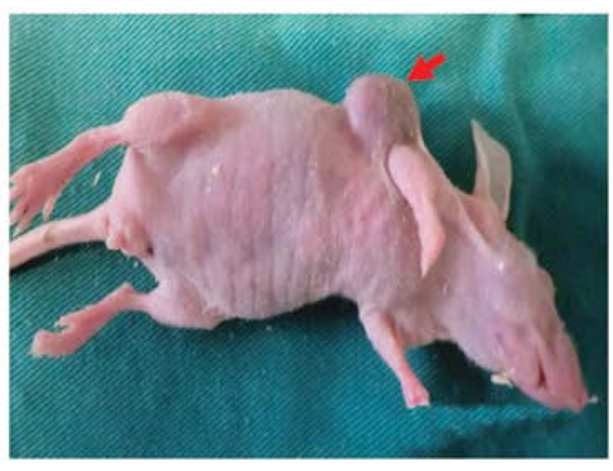

B

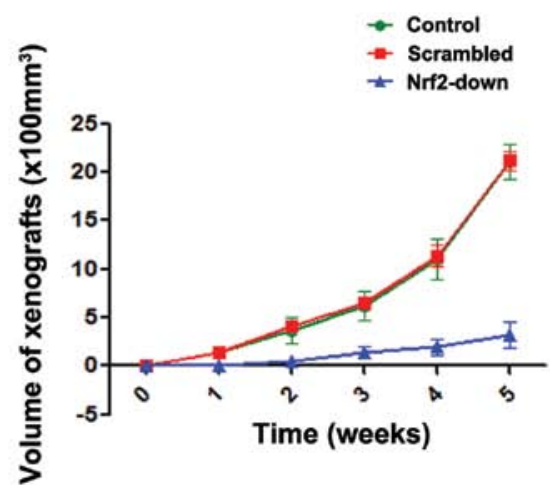

C

H\&E
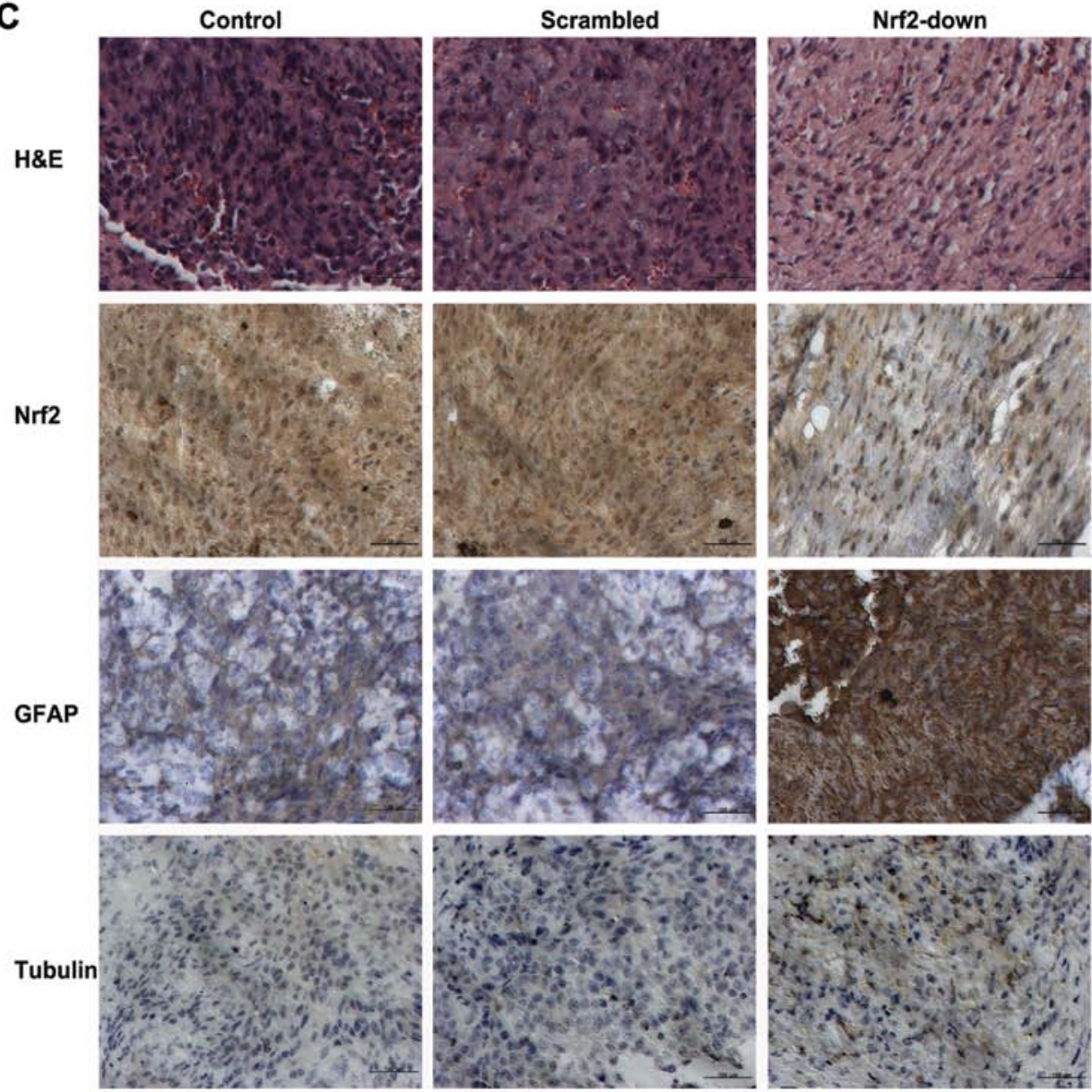

Figure 6. Knockdown of Nrf2 attenuates tumor growth and induces the differentiation of GSCs in vivo. (A) A tumor in a nude mouse (arrow). (B) Curve of tumor volume in nude mice. (C) H\&E staining and immunohistochemistry of tumors in nude mice. Tumor cells in Nrf2-down group were more mature and expressed higher GFAP and $\beta$ III-tubulin. H\&E, hematoxylin and eosin; Nrf2, nuclear factor erythroid 2-related factor 2; GFAP, glial fibrillary acidic protein.

of downstream gene HO-1 ( $\mathrm{P}=0.002)$ and NQO-1 $(\mathrm{P}=0.002)$ which were regulated by $\mathrm{Nrf2}$, were also decreased (Fig. $5 \mathrm{~B}$ ).

Knockdown of Nrf2 induced the differentiation of GSCs in vivo. GSCs from the control, scrambled and Nrf2-knockdown groups were inoculated subcutaneously to nude mice separately to observe the growth rate of GSCs in vivo (Fig. 6A). The tumor volume of GSCs from the Nrf2-down group was significantly smaller than the other two groups $(2,100 \pm 74.5$ vs. $2,117 \pm 104.6$ vs. $316 \pm 54.3 \mathrm{~mm}^{3}, \mathrm{P}<0.001$ ) (Fig. $6 \mathrm{~B}$ ).

Further H\&E staining showed the lower nuclear density and higher cytoplasm proportion in the Nrf2 downregulated group (Fig. 6C). Immunohistochemistry staining of xenograft tumor tissues demonstrated increased GFAP positive cells in the Nrf2 downregulated group, while $\beta$ III-tubulin was not significantly increased (Fig. 6C).

\section{Discussion}

Glioblastoma is the most prevalent and malignant primary central nervous system tumor (1). Standard therapy includes surgical incision, chemotherapy and radiotherapy. In spite of aggressive treatment, prognosis remains poor and many 
patients manifest resistance to traditional chemotherapy and radiotherapy. Studies have shown that the therapeutic resistance may be due to a crucial subpopulation of glioma stem cells (GSCs) $(22,23)$. GSCs present low status of differentiation and display high proliferation and high rates of self-renewal. Previous studies showed GSCs in glioblastoma conferred tumorigenic potential and resistance to chemotherapy and radiotherapy $(11,12,24)$. As a result, finding an effective method to change the differentiation status of GSCs, inducing it to differentiate into more mature ones, is key in eliminating the GSCs and enhancing the therapeutic sensitivity to chemotherapy and radiotherapy, thereby improving the prognosis of patients with glioblastoma.

Nrf2 is described as a central orchestrator of antioxidant and detoxifying genes involved in phase II detoxication enzymes and antioxidant stress enzymes $(13,25)$.

The role of Nrf2 was found in several types of tumors, such as non-small cell lung cancer, glioma, bladder carcinoma and hepatocarcinoma (26-28). Nrf2 can protect tumors from chemotherapy and radiotherapy $(19,20)$. In general, Nrf2 is highly expressed in cells with low status of differentiation. In our previous study, GSCs expressed much higher Nrf2 than glioblastoma and displayed a lower status of differentiation $(29,30)$. Pathological research demonstrated the expression of Nrf2 decreases with the differentiation increase in glioblastoma (30). This evidence indicates that $\mathrm{Nrf} 2$ may play an important role in regulating the differentiation of GSCs.

In this study, the GSCs separated from glioblastoma had a low status of differentiation, and expressed a high level of Nrf2. When we exogenously induced the differentiation of GSCs, the expression of Nrf2 decreased with the differentiation process. This indicates that $\mathrm{Nrf} 2$ is important in retaining the stem cell characteristics and maintaining the low status of differentiation in GSCs.

Furthermore, we knocked down Nrf2 in GSCs by shRNA transformed by lentivirus. Following Nrf2 knockdown, sphere-like growing was inhibited in the early stage of culture. Over time, the GSCs transformed to mature cells, and the astrocytoma surface marker GFAP and the neuronal surface marker $\beta$ III-tubulin expression increased. In vivo study showed similar results. Following Nrf2 knockdown, the growth rate of GSCs in vivo was significantly reduced, and the differentiation of xenografted tumor cells was improved. These results suggest that Nrf2 is a key factor inhibiting the differentiation of GSCs, and knockdown of Nrf2 can promote the differentiation process both in vitro and in vivo.

In addition, the expression of NQO-1 and HO-1 in GSCs was decreased after Nrf2 knockdown. Both NQO-1 and HO-1 are important in eliminating the reactive oxygen species (ROS) in cells (31-33). In previous studies, ROS was shown to play a key role in many pathological processes in cancer (34). The decrease of NQO-1 and HO-1 in this study indicates that Nrf2 may keep the low status of differentiation by inhibiting the ROS in GSCs. However, the molecular mechanism of the relationship between ROS and Nrf2 in the differentiation of GSCs requires further investigation.

Collectively, Nrf2 plays a critical role in maintaining the low status of differentiation of GSCs, and inhibition of Nrf2 can induce the differentiation, thereby providing a valuable therapeutic target for the elimination of GSCs. Efficient therapy blocking molecular target of Nrf 2 may benefit patients with glioblastoma.

\section{Acknowledgements}

The authors thank Dr Feng Genbao and Dr He Jin for the technical assistance. This study was supported by Grants from the National Natural Science Foundation of China (Nos. 81070974 and 81271377), the Jiangsu Provincial Key Subject (no. X4200722) and the Jinling Hospital of Nanjing, China (no. 2010Q017).

\section{References}

1. Van Meir EG, Hadjipanayis CG, Norden AD, Shu H, Wen PY and Olson JJ: Exciting new advances in neuro-oncology: the avenue to a cure for malignant glioma. CA Cancer J Clin 60: 166-193, 2010.

2. Yang L, Lin C, Wang L, Guo H and Wang X: Hypoxia and hypoxia-inducible factors in glioblastoma multiforme progression and therapeutic implications. Exp Cell Res 318: 2417-2426, 2012.

3. Reya T, Morrison SJ, Clarke MF and Weissman1 IL: Stem cells, cancer, and cancer stem cells. Nature 414: 105-111, 2001.

4. Singh SK, Clarke ID, Terasaki M, Bonn VE, Hawkins C, Squire J and Dirks PB: Identification of a cancer stem cell in human brain tumors. Cancer Res 63: 5821-5828, 2003.

5. Fan X, Salford LG and Widegren B: Glioma stem cells: evidence and limitation. Semin Cancer Biol 17: 214-218, 2007.

6. Jin X, Jin X, Jung JE, Beck S and Kim H: Cell surface Nestin is a biomarker for glioma stem cells. Biochem Biophys Res Commun 433: 496-501, 2013.

7. He J, Shan Z, Li L, Liu F, Liu Z, Song M and Zhu H: Expression of glioma stem cell marker CD133 and O6-methylguanine-DNA methyltransferase is associated with resistance to radiotherapy in gliomas. Oncol Rep 26: 1305-1313, 2011.

8. Park D, Xiang AP, Mao FF, et al: Nestin is required for the proper self-renewal of neural stem cells. Stem Cells 28: 2162-2171, 2010.

9. Park DM and Rich JN: Biology of glioma cancer stem cells. Mol Cells 28: 7-12, 2009

10. Shmelkov SV, St Clair R, Lyden D and Rafii S: AC133/CD133/ Prominin-1. Int J Biochem Cell Biol 37: 715-719, 2005.

11. Dean M, Fojo T and Bates S: Tumour stem cells and drug resistance. Nat Rev Cancer 5: 275-284, 2005.

12. Johannessen TC, Bjerkvig R and Tysnes BB: DNA repair and cancer stem-like cells - potential partners in glioma drug resistance. Cancer Treat Rev 34: 558-567, 2008.

13. Alam J and Stewart D: Nrf2, a Cap'n'Collar transcription factor, regulates induction of the heme oxygenase-1 gene. J Biol Chem 274: 26071-26078, 1999.

14. Kensler TW, Wakabayashi N and Biswal S: Cell survival responses to environmental stresses via the Keap1-Nrf2-ARE pathway. Annu Rev Pharmacol Toxicol 47: 89-116, 2007.

15. Kim HJ, Zheng M, Kim SK, Cho JJ, Shin CH, Joe Y and Chung HT: CO/HO-1 induces NQO-1 expression via Nrf2 activation. Immune Netw 11: 376-382, 2011.

16. Piao MS, Choi JY, Lee DH, Yun SJ, Lee JB and Lee SC: Differentiation-dependent expression of NADP $(\mathrm{H})$ :quinone oxidoreductase-1 via NF-E2 related factor-2 activation in human epidermal keratinocytes. J Dermatol Sci 62: 147-153, 2011.

17. Ji L, Li H, Gao P, Shang G, Zhang DD, Zhang N and Jiang T: Nrf2 pathway regulates multidrug-resistance-associated protein 1 in small cell lung cancer. PLoS One 8: e63404, 2013.

18. Tsai JJ, Dudakov JA, Takahashi K, et al: Nrf2 regulates haematopoietic stem cell function. Nat Cell Biol 15: 309-316, 2013.

19. Wang XJ, Sun Z, Villeneuve NF, et al: Nrf2 enhances resistance of cancer cells to chemotherapeutic drugs, the dark side of $\mathrm{Nrf} 2$. Carcinogenesis 29: 1235-1243, 2008.

20. Lau A, Villeneuve NF, Sun Z, Wong PK and Zhang DD: Dual roles of Nrf2 in cancer. Pharmacol Res 58: 262-270, 2008.

21. Zhu J, Wang H, Sun Q, et al: Nrf2 is required to maintain the self-renewal of glioma stem cells. BMC Cancer 13: 380, 2013.

22. Vescovi AL, Galli R and Reynolds BA: Brain tumour stem cells. Nat Rev Cancer 6: 425-436, 2006. 
23. Venere M, Fine HA, Dirks PB and Rich JN: Cancer stem cells in gliomas: identifying and understanding the apex cell in cancer's hierarchy. Glia 59: 1148-1154, 2011.

24. Stiles CD and Rowitch DH: Glioma stem cells: a midterm exam. Neuron 58: 832-846, 2008.

25. Cullinan SB, Zhang D, Hannink M, et al: Nrf2 is a direct PERK substrate and effector of PERK-dependent cell survival. Mol Cell Biol 23: 7198-7209, 2003.

26. Iida K, Itoh K, Kumagai Y, et al: Nrf2 is essential for the chemopreventive efficacy of oltipraz against urinary bladder carcinogenesis. Cancer Res 64: 6424-6431, 2004.

27. Singh A, Misra V, Thimmulappa RK, et al: Dysfunctional KEAP1-NRF2 interaction in non-small-cell lung cancer. PLoS Med 3: e420, 2006.

28. Ikeda H, Nishi S and Sakai M: Transcription factor Nrf2/MafK regulates rat placental glutathione $\mathrm{S}$-transferase gene during hepatocarcinogenesis. Biochem J 380: 515-521, 2004.

29. Pan H, Wang H, Zhu L, Wang X, Cong Z, Sun K and Fan Y: The involvement of Nrf2-ARE pathway in regulation of apoptosis in human glioblastoma cell U251. Neurol Res 35: 71-78, 2013.
30. Ji XJ, Chen SH, Zhu L, et al: Knockdown of NF-E2-related factor 2 inhibits the proliferation and growth of U251MG human glioma cells in a mouse xenograft model. Oncol Rep 30: 157-164, 2013.

31. Park EJ, Lim JH, Nam SI, Park JW and Kwon TK: Rottlerin induces heme oxygenase-1 (HO-1) up-regulation through reactive oxygen species (ROS) dependent and PKC delta-independent pathway in human colon cancer HT29 cells. Biochimie 92: 110-115, 2010.

32. Kim JE, Kang YJ, Lee KY and Choi HC: Isoproterenol inhibits angiotensin II-stimulated proliferation and reactive oxygen species production in vascular smooth muscle cells through heme oxygenase-1. Biol Pharm Bull 32: 1047-1052, 2009.

33. Hsieh HL, Wang HH, Wu CY and Yang CM: Reactive oxygen species-dependent c-Fos/activator protein 1 induction upregulates heme oxygenase-1 expression by bradykinin in brain astrocytes. Antioxid Redox Signal 13: 1829-1844, 2010.

34. DeNicola GM,Karreth FA,Humpton TJ,et al: Oncogene-induced Nrf2 transcription promotes ROS detoxification and tumorigenesis. Nature 475: 106-109, 2011. 